\title{
A Historical Review of U.S. Contributions to the Atomic Redefinition of the SI Second in 1967
}

\begin{abstract}
Michael A. Lombardi
National Institute of Standards and Technology, Boulder, CO 80305, USA

lombardi@nist.gov

This paper was written to commemorate the $50^{\text {th }}$ anniversary of the atomic redefinition of the second in the International System (SI) in 1967. It focuses on the work of individuals and organizations in the United States who made significant contributions to the redefinition of the SI second and helped to establish the era of atomic timekeeping.
\end{abstract}

Key words: atomic clock; cesium; frequency; second; time.

Accepted: May 25, 2017

Published: June 1, 2017

https://doi.org/10.6028/jres.122.029

\section{Introduction}

The second, the base unit of time interval, is one of the seven base units in the International System (SI). Because time interval and its reciprocal, frequency, can be measured with more resolution and less uncertainty than any other physical quantity, the second holds a place of preeminence in the world of metrology. The precise realization of the SI second that we benefit from today was made possible by the development of atomic frequency standards and clocks; devices that fundamentally changed the way that time is measured and kept. Before atomic clocks, the second was defined by dividing astronomical events, such as the solar day or the tropical year, into smaller parts. This permanently changed in 1967, when the SI second was redefined as the duration of 9192631770 periods of the electromagnetic radiation that causes ground state transitions in the cesium atom [1]. The new definition meant that seconds were now measured by counting oscillations of electric fields that cause atoms to change state, and minutes and hours were now multiples of the second rather than divisions of the day.

The benefits of atomic timekeeping to modern society have been immense and are difficult to overestimate. Many technologies that we now take for granted, such as global navigation satellite systems, mobile telephones, and the "smart grids" that provide our electric power, depend upon atomic clock accuracy. This makes it easy to forget that the era of atomic timekeeping began less than an average lifetime ago. The current year (2017) marks a half-century since the International System of Units (SI) adopted a definition of the second based on an atomic transition [1]. To commemorate this $50^{\text {th }}$ anniversary, this paper provides a historical review of work that contributed to the atomic definition of the SI second in 1967. Its primary focus is on work conducted in the United States of America.

\section{The First Suggestion of Atomic Clocks and Early Research}

The possibility of an atomic clock was first suggested in Europe in the 19th century. James Clerk Maxwell, a Scottish physicist, was likely the first person to recognize that atoms could be used to keep time. In an era during which the Earth's rotation was the world's timekeeping reference, Maxwell 
remarkably suggested to Peter Guthrie Tait (a childhood friend) and William Thomson (later to be known as Lord Kelvin) that the "period of vibration of a piece of quartz crystal of specified shape and size and at a stated temperature" would be a better absolute standard of time than the mean solar second, but it would still depend "essentially on one particular piece of matter, and is therefore liable to all the accidents, etc. which affect so-call National Standards however carefully they may be preserved, as well as the almost insuperable practical difficulties which are experience when we attempt to make exact copies of them." [2] Atoms would work even better as a natural standard of time. As Thomson and Tait wrote in their Elements of Natural Philosophy, first published in 1879:

"The recent discoveries ... indicate to us natural standard pieces of matter such as atoms of hydrogen or sodium, ready made in infinite numbers, all absolutely alike in every physical property. The time of vibration of a sodium particle corresponding to any one of its modes of vibration is known to be absolutely independent of its position in the universe, and will probably remain the same so long as the particle itself exists." [2, 3]

It was to be some six decades after these prophetic mentions of atomic clocks before the first experiments were performed. The experiments were finally made possible by the rapid advances in quantum mechanics and microwave electronics that took place before, during, and after World War II. Most of the fundamental concepts that would eventually lead to an atomic clock were developed by Isidor Isaac Rabi and his colleagues at Columbia University in New York City, beginning in the 1930s [4, 5]. Born in 1898 in Rymanów, Galicia, then part of Austria-Hungary (now Poland), Rabi emigrated to the United States as an infant, grew up in New York City, and went on to attend Columbia University. After initially studying electrical engineering and chemistry, he eventually received a doctorate in physics. In 1929, he joined the Columbia faculty. Shortly afterward, he built a molecular beam apparatus, and his new molecular beam laboratory soon began to attract other talented young physicists who would go on to play key roles in atomic clock history, including Sidney Millman, Jerrold Zacharias, Polykarp Kusch, and Norman Ramsey.

Rabi invented the atomic and molecular beam resonance method of observing atomic spectra in 1937, groundbreaking work for which he would receive the Nobel Prize in physics in 1944. As early as 1939, he had informally discussed applying his molecular beam magnetic resonance technique as a time standard with scientists at the National Bureau of Standards (NBS), then located in Washington, D.C. However, World War II soon followed, and Rabi, like most scientists of that era, halted his own research to assist in the war effort. During the war, he led a group of scientists at the Massachusetts Institute of Technology (MIT) in Cambridge, Massachusetts, who helped in the development of radar. During the final year of the war, Rabi publicly discussed the possibility of atomic clocks for the first time in a lecture given to the American Physical Society and the American Association of Physics Teachers on January 20, 1945 [6]. The following day, the New York Times covered the story (Fig. 1) with a lead sentence referring to the "most accurate clock in the universe." [7]

The New York Times story referred to a "cosmic pendulum," a metaphorical reference acknowledging that the basic principles of atomic clocks were straightforward and were already well understood by the early researchers. In short, because all atoms of a specific element are identical (ignoring isotopic differences), they should undergo a transition in their energy level when stimulated with electromagnetic radiation at a specific frequency. This meant that an atom could potentially serve as a nearly perfect resonator for a clock. The frequency that caused the energy transition could be used as a standard of frequency, and the periods of this frequency could be counted to define a standard for time interval. It easily followed that a clock referenced to an atomic resonator would be far more accurate than previous clocks that were based on mechanical oscillations of a pendulum or a quartz crystal.

Although the concept of an atomic clock was seemingly simple, its implementation was exceedingly difficult, particularly with the technology that existed in the 1940s. Atomic transitions occurred in the microwave region, at much higher frequencies than had previously been measured, and systems and electronics were needed that could measure these microwave frequencies. Determining the resonance frequency of a specific element was necessary so that it could be related to the second, the base unit of time interval. The next step would be to use the atomic resonance frequency to control the frequency of a 
physical oscillator, such as a quartz crystal oscillator, by locking it to atomic resonance. Finally, to make the output of an atomic clock usable, the frequency of the locked quartz oscillator would need to be divided into frequencies low enough to use for timekeeping.

\section{'COSMIC PENDULLW' FOR CLOCK PLANNED}

\section{Radio Frequencies in Hearts of Atoms Would Be Used in Most Accurate of Timepieces \\ DESIGN TERMED FEASIBLE}

\section{Prof. I. I. Rabi, 1944 Nobel Prize Winner, Tells of Newest Developments}

By WILLIAM L. LAURENCE

Blueprints for the most accurate clock in the universe, tuning in on radio freguencies in the hearts of atoms and thus beating in harmony with the "cosmic pendulum," were outlined yesterday at the annual New York meeting of the American Physical Society, at Columbia University, by Prof. I. I. Rabi, who delivered the Richtmyer Memorial Lecture under the auspices of the American Association of Physics Teachers.

Fig. 1. The first public suggestion of an atomic clock, New York Times, January 21, 1945, p. 34 (first paragraph of article).

The first trick was to be able to measure the resonance frequency of an atom, which is derived from its quantized energy levels. The laws of quantum mechanics dictate that the energies of a bound system, such as an atom, have certain discrete values. An electromagnetic field generated at a specific frequency can boost an atom from one energy level to a higher one, or an atom at a high-energy level can drop to a lower level by emitting energy. The resonance frequency $\left(f_{0}\right)$ of an atomic oscillator is the difference between the two energy levels, $E_{1}$ and $E_{2}$, divided by Planck's constant, $h$ :

$$
f_{\mathrm{o}}=\frac{E_{2}-E_{1}}{h} \text {. }
$$

The energy absorbed or emitted by the atoms is spread across a small frequency range that surrounds $f_{0}$, and therefore it does not exist at $f_{\mathrm{o}}$ alone. This spread of frequencies, $\Delta f_{\mathrm{a}}$, is known as the resonance width, or linewidth. The ratio of the resonance frequency to the resonance width is known as the quality factor, $Q$, where

$$
Q=\frac{f_{\mathrm{o}}}{\Delta f_{\mathrm{a}}} .
$$

Measuring the atomic linewidth was another challenging task faced by early researchers. However, because their resonance frequencies were so high, it was obvious that an atomic clock, even with a broad 
linewidth, would have a much higher $Q$ than any previous clock and would therefore have a potentially huge advantage in both accuracy and stability.

It seems likely that Rabi and his colleagues expected the energy transition in cesium $\left({ }^{133} \mathrm{Cs}\right)$ atoms to be the resonator for the first atomic clock [8]. Millman and Kusch (who, like Rabi, would become a Nobel Laureate in 1955), had first measured the cesium resonance frequency at Columbia in 1940, estimating the frequency of the hyperfine transition as 9191.4 megacycles. This was relatively close to the number that would later define the second [9]. Fate would intervene, however, and the first atomic clock was not based on cesium, but instead was an ammonia absorption device built at NBS.

\section{The First Atomic Clock, 1948}

Harold Lyons was certainly not the first scientist to perform spectroscopic frequency stabilization experiments or to engage in atomic clock research, but he and his colleagues at NBS were the first to build a working atomic clock. Born in Buffalo, New York, in 1913, Lyons was a graduate student at the University of Michigan (he obtained a Ph.D. in nuclear physics in 1939) when Rabi and his colleagues at Columbia first discussed employing their molecular beam resonance technique as a time standard. Lyons joined NBS in 1941 and became head of the microwave standards group in 1948. There, he and his colleagues moved rapidly past the pure experimental stage and began constructing a working atomic clock. After a period of intense research and development beginning in the spring of 1948, the new clock (Fig. 2) was first operated on August 12, 1948, and was publicly demonstrated in January 1949 [10].

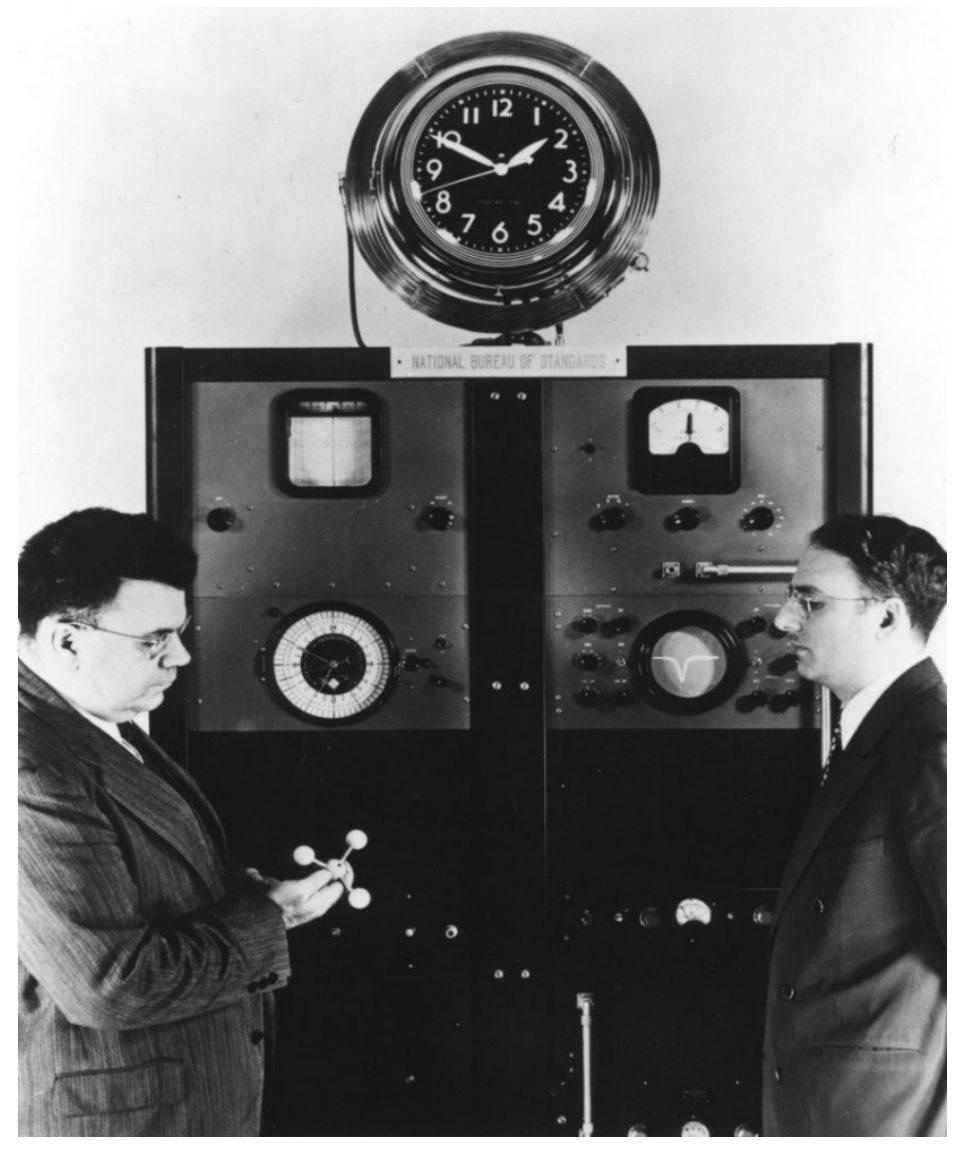

Fig. 2. Dr. Harold Lyons (right), inventor of the ammonia absorption cell atomic clock, observes, while Dr. Edward U. Condon, the director of the National Bureau of Standards, examines a model of the ammonia molecule (1949). The clock atop the equipment racks was driven by a $50 \mathrm{~Hz}$ signal obtained by dividing the signal from a quartz oscillator that was locked to the ammonia absorption frequency. However, its primary function was to let the world know that the invention was indeed a clock. 
The construction of an atomic clock in 1948 was a significant scientific and engineering accomplishment that required many obstacles to be overcome. Because he had chosen the absorption line of ammonia gas as the source of frequency, Lyon's first obstacle was measuring the inversion transition frequency, or what Lyons called, in keeping with Thomson and Tait [2], the "vibration" [11], of the atoms inside ammonia molecules. The ammonia gas was stored in an absorption cell, which was designed as a 30 $\mathrm{ft}$ long ( $\sim 9 \mathrm{~m}$ long) copper tube that was wrapped into a spiral. The measurement involved multiplying a $100 \mathrm{kHz}$ signal from quartz oscillator several times. The first stage consisted of a frequency multiplication chain built from low-frequency tubes that resulted in a frequency of about $270 \mathrm{MHz}$. The second stage consisted of a frequency multiplying klystron (a linear beam vacuum tube), wherein frequency was modulated by a $13.8 \mathrm{MHz}$ oscillator that multiplied the signal to about $2.983 \mathrm{GHz}$. Finally, the signal was multiplied to approximately $23.87 \mathrm{GHz}$ by use of a silicon crystal rectifier. This frequency was fed to the ammonia absorption cell and tuned until the signal reaching a silicon crystal detector at the end of the cell showed a spectral line (in the form of a dip, or inverted peak) when displayed on an oscilloscope (Fig. 3). The line indicated that the incoming frequency from the multiplication chain now matched the ammonia absorption frequency. When the line occurred, an electrical pulse was generated [11-14].

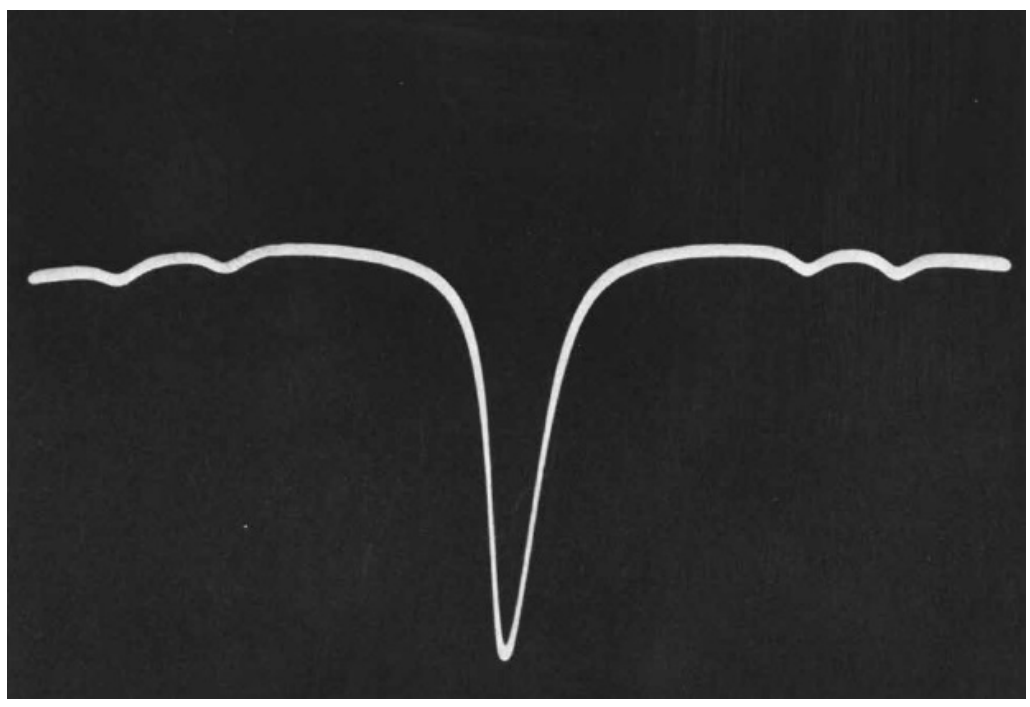

Fig. 3. The spectral line (shown on an oscilloscope) that occurred when the incoming microwave signal from the frequency multiplication chain matched the absorption frequency of the ammonia molecule.

The next obstacle faced by Lyons and his group was to develop a method to automatically adjust the quartz oscillator frequency until it matched the frequency of the ammonia absorption line. This was done by taking a $12.5 \mathrm{MHz}$ frequency from the quartz oscillator frequency multiplication chain and mixing it with the signal from the $13.8 \mathrm{MHz}$ frequency-modulated oscillator. When the frequency from the mixer matched its expected value, another electrical pulse was generated. The time interval between the two generated pulses (the pulse from the absorption cell and the pulse from the mixer) was continuously measured. If the time interval was increasing, it indicated that the quartz oscillator frequency was increasing, and vice versa. The time interval measurements were used to generate a control signal that adjusted the quartz oscillator whenever necessary to keep it locked to the ammonia absorption frequency $[11,13]$.

Figure 4 shows a strip chart where the quartz oscillator was locked to the ammonia absorption frequency from 5 p.m. to 8 a.m. Before and after this period, the quartz oscillator was "unlocked" (free running). Each small division on the strip chart represents a frequency variation of less than 1 part per 10 million $\left(1 \times 10^{-7}\right)$. 


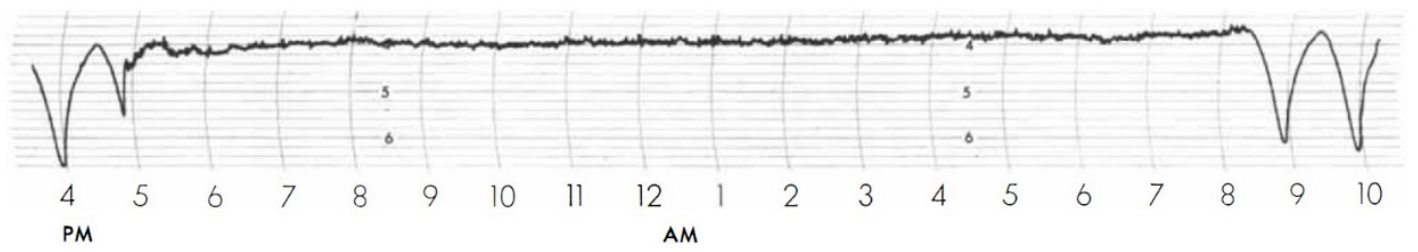

Fig. 4. A strip chart showing a period (from 5 p.m. to 8 a.m.) where the quartz oscillator was locked to the absorption frequency of ammonia.

A third obstacle involved dividing the locked quartz oscillator frequency into low frequencies that could be used for timekeeping [15]. The first atomic clock included dividers that produced two output signals, a $50 \mathrm{~Hz}$ signal that drove an ordinary synchronous motor clock, and a $1000 \mathrm{~Hz}$ signal that was used to drive a special synchronous motor clock that was designed for comparisons with astronomical time (then the world standard for timekeeping) to within 5 ms. Figure 5 is a simplified block diagram showing how the clock worked.

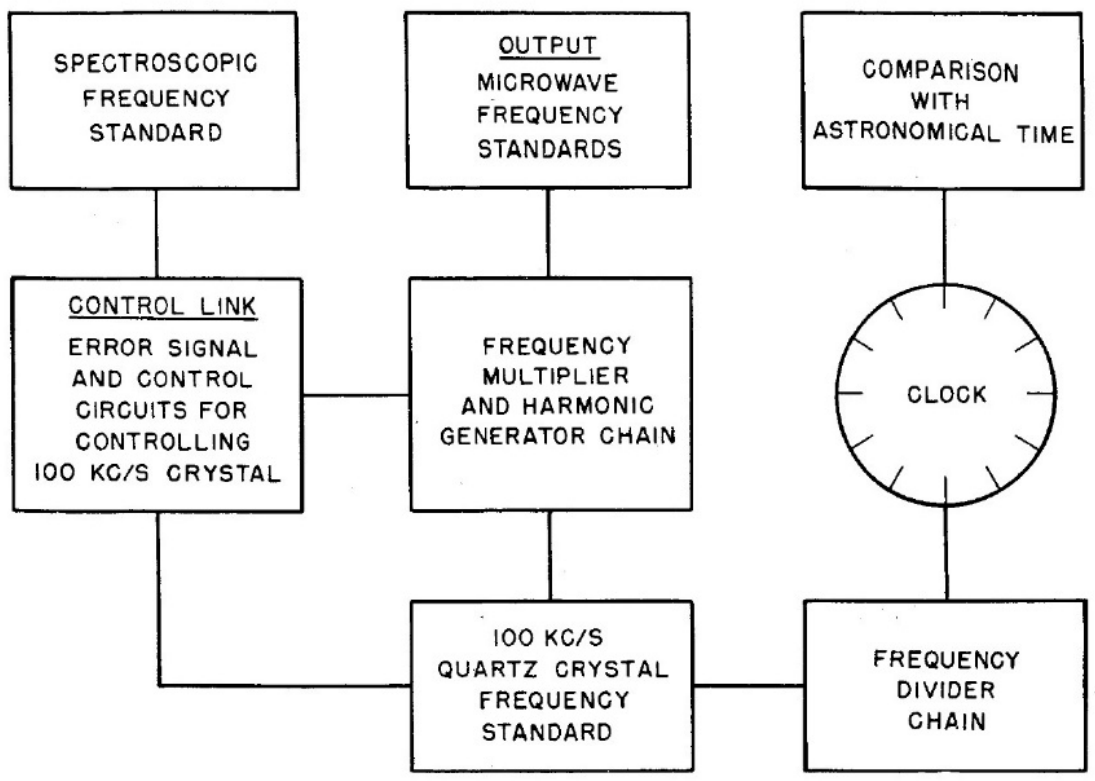

Fig. 5. A simplified block diagram of the first atomic clock.

A final technical obstacle faced by Lyons was his quest to make his ammonia clock more accurate by increasing the quality factor, $Q$, as previously discussed and shown in Eq. (2). It was already known that oscillators with higher $Q$ values would be potentially more stable and accurate, so it was desirable to increase $Q$ as much as possible, either by using an atomic transition where the frequency was as high as possible, or by making the linewidth as narrow as possible. In the case of the ammonia clock, the resonance frequency was high $(23.8 \mathrm{GHz})$, but the resonance width, or the range of frequencies over which the oscillator could resonate, represented by the width of the line in the absorption frequency shown in Fig. 3, was not especially narrow. Lyons and his colleagues had little success when attempting to reduce the linewidth. Thus, the actual $Q$ factor of the ammonia spectrum line was typically measured in a range from 50000 to 500 000, approximating that of the best quartz crystal oscillators of that era, although it was much more constant and stable [12]. 
In a metrological sense, Lyons and his colleagues had advantages over university researchers, because NBS already had several decades of experience in maintaining, disseminating, and measuring standard frequency signals. This allowed Lyons to accurately measure his new clock against the national standard of frequency, the quartz oscillators operated by NBS that were periodically calibrated against the astronomical time standards maintained by the U.S. Naval Observatory. He quickly learned from these measurements that the potential accuracy of the ammonia clock was limited. Two versions of the ammonia clock were built, with the best reported accuracy about $2 \mathrm{~ms} /$ day $\left(2 \times 10^{-8}\right)$. Work on a third version was halted when it became apparent that cesium beam techniques were likely to provide a significant increase in accuracy. Even though the cesium resonance frequency was much lower $(\sim 9.2 \mathrm{GHz})$, there were numerous options available for decreasing the linewidth, leading to $Q$ factors that were much higher than those obtained with molecular absorption methods. Thus, by the early part of 1950, Lyons and his NBS colleagues had turned their attention away from ammonia clocks, and with some assistance from Kusch at Columbia University, had begun constructing a cesium beam clock [16].

\section{Cesium Atomic Clocks}

Cesium had several characteristics that made it suitable for use in an atomic clock. A silvery-white ductile metal, cesium is the softest element listed on Mohs hardness scale (0.2 MPa) and one of the few elemental metals that becomes a liquid at near room temperature $\left(28.4^{\circ} \mathrm{C}\right)$; only mercury has a lower known melting point. Cesium atoms are also relatively heavy and thus move at a relatively slow speed of about $130 \mathrm{~m} / \mathrm{s}$ at room temperature in an atomic beam. This allows cesium atoms to be observed for a longer period than hydrogen atoms, for example, which travel much faster, about $1600 \mathrm{~m} / \mathrm{s}$, at room temperature. Cesium also has a higher resonance frequency $(\sim 9.2 \mathrm{GHz})$ than other atoms that were later utilized in atomic clocks, such as rubidium $(\sim 6.8 \mathrm{GHz})$ and hydrogen $(\sim 1.4 \mathrm{GHz})$.

The first cesium clock built by Lyons and Jesse Sherwood at NBS utilized Rabi’s magnetic resonance technique. Sherwood reported initial results at a 1952 meeting of the American Physical Society [17], but the resonance width of the clock was too wide ( $\sim 30 \mathrm{kHz}$ ) to serve as an accurate time standard (the $Q$ was only 300000 ). Once cesium was selected as the atom of choice, the resonance frequency could not be changed, so the focus turned to making the resonance width as narrow as possible. Narrowing the resonance width required a better way of interrogating the cesium atoms.

Rabi's original magnetic resonance technique interrogated the atoms with one microwave pulse. Methods were developed to increase the $Q$ by applying longer microwave pulses, but these schemes did not work as expected due to subtle technical side effects; the long interaction period between the atoms and microwave field subjected the frequency of the clock to Doppler shifts and other uncertainties. A breakthrough occurred in 1949, when Norman Ramsey, a former student of Rabi who was then a professor at Harvard University, invented the separated oscillatory field method, an improvement that would be critically important to future atomic clock designs. Ramsey's new method interrogated the atoms with two short microwave pulses, separated by some distance in an atomic beam. Applying the oscillating field in two steps accomplished the goal of narrowing the resonance width. It also reduced the sensitivity to microwave power fluctuations and magnetic fields by factors of 10 to 100 or more, and it eliminated most of the Doppler effect. In short, Ramsey made it possible to build much more accurate atomic clocks $[5,18]$. Some four decades later, in 1989, Ramsey received a Nobel Prize in physics for this work.

The NBS team quickly redesigned their clock to employ Ramsey's new technique of separated oscillating fields by applying microwave pulses in separated regions along an atomic beam. This reduced the resonance width to just $300 \mathrm{~Hz}$, increasing the $Q$ to about 30 million. Encouraged by these results, Lyons predicted that the clock would eventually be accurate to $1 \times 10^{-10}$, meaning that it would be able to keep time to within about $10 \mu \mathrm{s} /$ day [19]. His prediction eventually came true, but it happened much later than expected. In 1953, NBS interrupted their atomic clock program, a decision made partially for budgetary and political reasons and partially because the agency elected to focus on other areas. Sherwood had resigned in 1952 [10], and Lyons moved to the new NBS laboratories in Boulder, Colorado, in 1954. A year later, he left NBS entirely, accepting a position at Hughes Aircraft Company in Culver City, California. 
The cesium clock was disassembled and moved from Washington, D.C., to Boulder, where it was reassembled by a new team of researchers that was led by Richard Mockler and included Roger Beehler and Chuck Snider. It eventually became a working time standard in 1958. Then known as NBS-1 (Fig. 6), the clock finally achieved Lyon's predicted accuracy [20, 21].

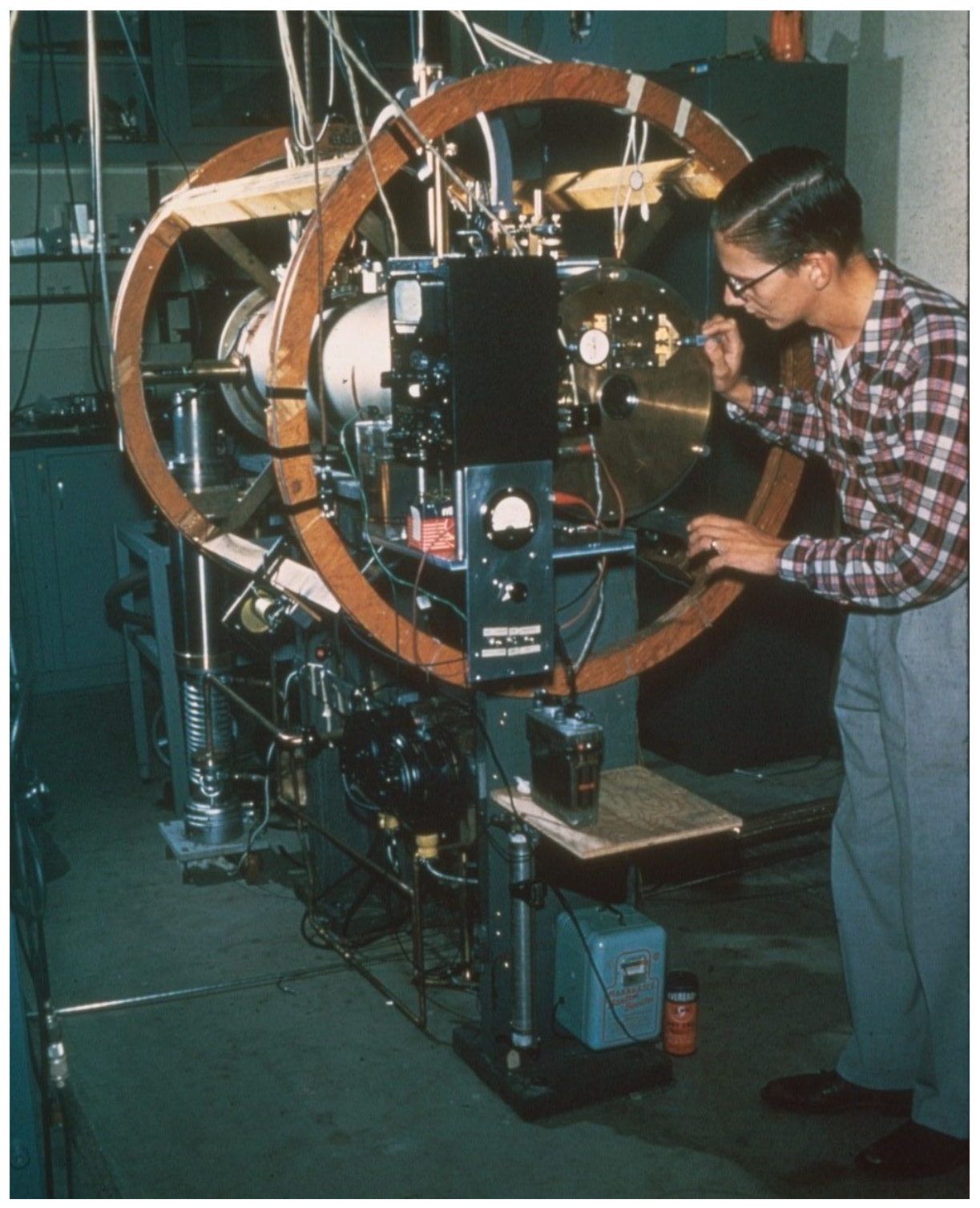

Fig. 6. Roger Beehler of NBS with NBS-1.

The length of the microwave interrogation cavity (commonly known as the Ramsey cavity) in NBS-1 was $55 \mathrm{~cm}$, and its linewidth, as previously noted, was about $300 \mathrm{~Hz}$ [22]. It was designated as the U.S. national standard for frequency in the spring of 1959, when its accuracy was reported as $8.5 \times 10^{-11}$ [23]. On January 1, 1960, it was replaced by NBS-2 as the national primary frequency standard, although it continued to be compared to NBS-2 as a secondary standard until 1962, when it had reached a reported accuracy of $1 \times 10^{-11}$ [24]. Ironically and perhaps appropriately, Ripley's Believe it or Not, a popular syndicated cartoon featured in U.S. newspapers, credited Lyons with the invention of the atomic clock in 1953, but the drawing was not of the ammonia clock, but rather of the cesium beam clock that would later become NBS-1 (Fig. 7). 


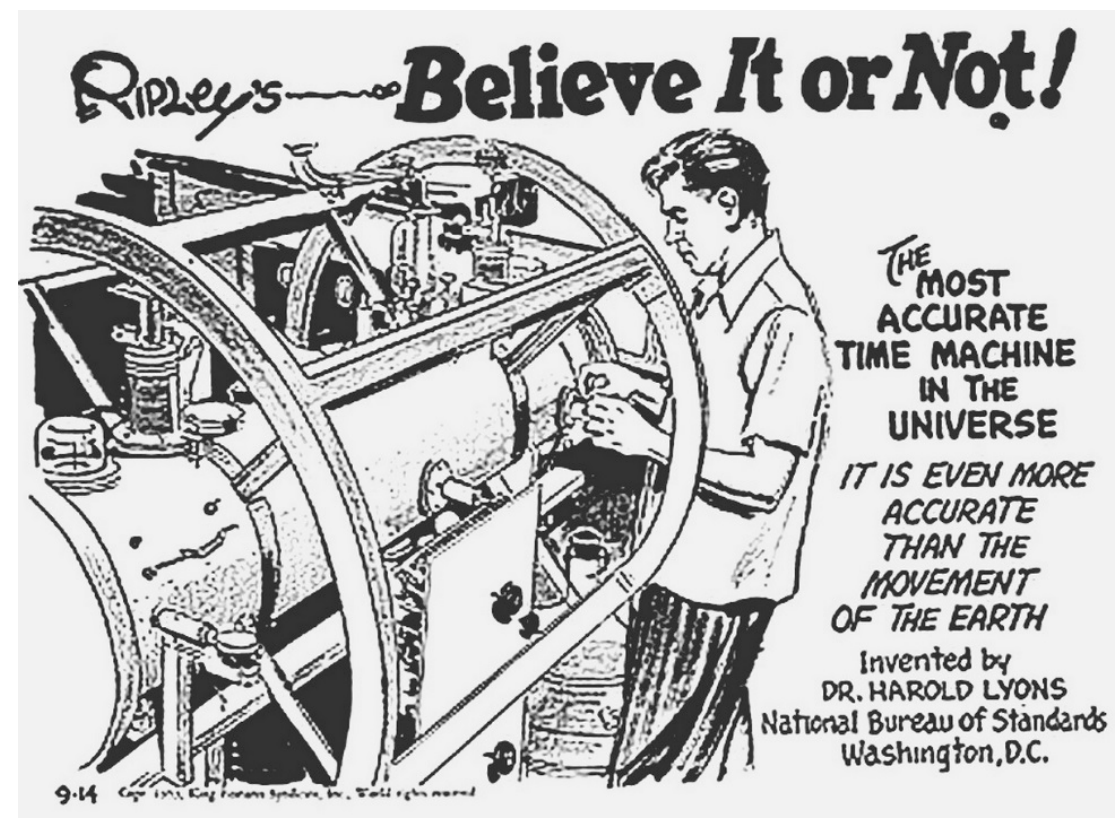

Fig. 7. A 1953 cartoon featuring the first cesium clock developed at NBS.

The disruption of the atomic clock program and a work stoppage of at least several years caused NBS to lose their large early lead in the cesium clock race, and the National Physical Laboratory (NPL) in the United Kingdom became the clear winner. The NPL effort was led by Louis Essen, who had previously worked on quartz clocks and measurements of the speed of light before becoming interested in atomic timekeeping. Beginning in 1949, Essen made several trips to the United States, where he met with early atomic clock researchers, including Rabi and his colleagues at Columbia and Jerrold Zacharias of MIT. Essen was anxious to apply their work to timekeeping and appealed to Sir Edward Bullard, the director of NPL, to start a new program designed to build an atomic clock. After several delays, work on a cesium clock began at NPL in 1953, the same year when work was essentially stopped at NBS, and it progressed rapidly enough for a working time standard to be placed in operation in June 1955. The linewidth was 340 $\mathrm{Hz}$, and the original reported accuracy was $1 \times 10^{-9}(100 \mu \mathrm{s} /$ day) $[25,26]$. The NPL cesium clock then famously played a key role in the experiment that led to the atomic definition of the SI second, as discussed in the next section.

\section{Defining the Atomic Second by Comparison to Ephemeris Time}

As noted in the introduction, prior to the invention of atomic clocks, the second was defined by dividing the period of an astronomical event into a shorter time interval. For example, the second was once defined by dividing the average period of one revolution of Earth on its axis. The mean solar second was equal to 1/86 400 of the mean solar day. To create a more stable unit of time interval, the second was redefined in 1956 as 1/31 556925.9747 of the tropical year 1900, a time interval known as the ephemeris second. The ephemeris second was indeed more stable than the mean solar second, but it was nearly impossible to use as a time reference in a laboratory, and was thus of little use to metrologists or engineers. In retrospect, it seems unfortunate that another astronomical definition of the second was accepted during a period when working atomic clocks were already being demonstrated [27, 28]. A clean transition from the mean solar second to the atomic second would certainly have made more sense. Doomed from the start, the ephemeris second would be easy for historians to dismiss had it not served as the comparison reference for the definition of the atomic second. 
Ephemeris time was determined by measuring the position of the Moon with respect to several surrounding stars. The most accurate Moon observations had been recorded at the U.S. Naval Observatory (USNO) in Washington, D.C., by the astronomer William Markowitz. Born in Austria in 1907, Markowitz's family emigrated to the United States when he was three years old. He obtained a Ph.D. in astronomy from the University of Chicago in 1931, and he worked at USNO from 1936 until 1966. By 1952, Markowitz was performing Moon observations with a sophisticated dual-rate Moon camera of his own design $[28,29]$.

Collecting Moon observations was a tedious task and results were obtained very slowly. Bullard, the NPL director, wrote in 1955 that it would take four years of Moon observations to determine time with the same accuracy as their new cesium clock. He also noted that "atomic clocks will be improved, probably by a greater factor than the astronomical determinations," which in retrospect was a considerable understatement [30]. To Bullard and many others, it was already clear when the ephemeris second became the SI second that atomic clocks represented the future of timekeeping.

In June 1955, due in part to the fact that there were no reliable atomic clocks then operating in the United States, NPL and USNO began collaborating on a program in which the goal was to determine the frequency of cesium with respect to the ephemeris second [31, 32]. It was a worthy goal, because redefining the second based on an atomic transition would allow large numbers of laboratories to simultaneously produce a physical realization of the second, which could in turn be used as the reference for other laboratory measurements. To achieve the goal, NPL and the USNO would need to compare their clocks and measure their differences, which they did from mid-1955 until the end of the first quarter of 1958, a period of 2.75 years. The USNO clock was based on a $2.5 \mathrm{MHz}$ quartz oscillator manufactured by Western Electric, ${ }^{1}$ which was steered to ephemeris time by applying corrections obtained with the dual-rate Moon camera. The NPL clock was based on their new cesium standard, which was now considered to be accurate to within $5 \times 10^{-10}$. Because the two clocks were on opposite sides of the Atlantic Ocean, the comparison could only be made by simultaneously comparing both clocks to radio signals that could be received at both laboratories, a measurement technique now known as common-view time transfer. Several time signal broadcast stations were involved in the measurement, including NBS radio station WWV, then located in Beltsville, Maryland, in the United States, and radio stations MSF and GBR, located in the United Kingdom [31].

To make the uncertainty of the measurement results as small as possible, four different solutions were calculated to determine the effects of using different sets of data. The final measurement result was the average of the four solutions, and it was published as 9192631770 cycles/s in August 1958, with an estimated measurement uncertainty of \pm 20 cycles/s. The measurement result applied to cesium in a zeromagnetic field and to ephemeris time at the beginning of 1957 [32]. The measurement uncertainty was limited not by the cesium standard, but rather due to the many complexities involved in measuring ephemeris time. Multiple sources of short-term noise, including the radio signals used in common-view time transfer and large uncertainties in the Moon camera itself, meant that the necessary resolution could only be achieved with very long averaging periods of at least 100 days. If we stop to consider the limitations of the technology that existed in the 1950s and the large number of complex steps that were required, the measurement results were remarkably accurate. As Leschiutta wrote in 2005:

"As a personal remark, taking into account the capabilities of the timing emissions at the moment, of the frequency standards available, of the inevitable scatter of the moon camera, and some other factors, not least the widespread use and abuse in 'touching' the piezo-oscillator, it is almost impossible to explain the accuracy of the Markowitz determination. Similar events, i.e. results surpassing the capabilities of the moment, are not uncommon in the history of science that sometimes is prone to accepting the intervention of a serendipity principle. The other possible explanation calls for a first class understanding of physics, coupled with scientific integrity." [31]

\footnotetext{
${ }^{1}$ Certain commercial equipment, instruments, or materials are identified in this paper for the purposes of illustration and description and to provide a historical narrative. Such identification does not imply recommendation or endorsement by the National Institute of Standards and Technology, nor does it imply that the materials or equipment identified are necessarily the best available for the purpose.
} 
The publication of the Markowitz/Essen measurement results [32] made it inevitable that an atomic time scale would eventually supplant the existing astronomical time scales [33, 34], yet still nearly a decade passed before the definition of the second was changed. At least part of the delay was because atomic standards other than cesium devices, specifically those based on hydrogen and thallium, were being considered as the basis for the redefinition. Jean Terrien, the director of the Bureau International des Poids et Mesures (BIPM), wrote in August 1967 that the definition had nearly been changed in 1964, but

"it was considered premature in 1964 to redefine the second in terms of the caesium frequency because the hydrogen maser was expected to offer, within a short while, a better base for such a redefinition.

Contrary to expectation, it happened that the hydrogen maser, though potentially slightly superior to the caesium beam frequency standard, progressed only slowly (one of its uncertainties comes from the wall shift), and that caesium standards were so much improved that an almost perfect confidence was acquired in their reliability, within the most severe present needs of precision. Though it was agreed that the improvement of other atomic frequency standards, using for example hydrogen or thallium atoms, ought to be pursued actively, the Comité Consultatif pour la Définition de la Seconde, at its meeting of 12-13 July 1967, recommended unanimously to the Comité International des Poids et Mesures not to wait any longer ...”[35]

Thus, in October 1967, the SI second was formally redefined by the Comité International des Poids et Mesures (CIPM) as:

"the duration of 9192631770 periods of the radiation corresponding to the transition between the two hyperfine levels of the ground state of the caesium 133 atom." [1]

As of 2017, the definition of the SI second remains the same, except for a slight amendment made in 1997. Calculations made by Wayne Itano of NBS in the early 1980s [36] revealed that blackbody radiation can cause noticeable frequency shifts in cesium standards, and his work eventually resulted in an addendum to the definition. The CIPM affirmed in 1997 that the definition refers to "a cesium atom at rest at a thermodynamic temperature of $0 \mathrm{~K}$." This meant that an optimal realization of the SI second requires the cesium atoms to be in a field-free environment where the temperature is absolute zero and where the atoms have no residual velocity.

When the atomic second became the SI second 1967, it was of course known that atomic time would continuously diverge from astronomical time, so periodic corrections would be needed to keep the new atomic time scale, called Coordinated Universal Time (UTC), in step with astronomical time. The term UTC had been used informally since the early 1960s, as the United Kingdom and United States had begun coordinating frequency adjustments made to their time scales beginning on January 1, 1960. Other countries followed suit, and in 1961, the Bureau International de l'Heure in Paris, France, began to oversee the international time coordination process [28]. Frequency adjustments continued to be used for almost five years after the new SI definition, but the procedure changed when the first leap second was inserted on June 30, 1972. Leap seconds are periodically added to UTC to keep it within $\pm 0.9 \mathrm{~s}$ of UT1, an astronomical time scale based on the mean solar second. Adding a leap second to UTC stops atomic time for one second to allow astronomical time to catch up.

The exact divergence between astronomical and atomic time is difficult to model or predict, but there are two general reasons why leap seconds are periodically needed. Reason one can be traced to the original Markowitz/Essen measurement; the atomic second was defined with respect to the ephemeris second, which then served as the SI second, and not with respect to the mean solar second, which forms the basis for UT1. Ephemeris seconds were thus already slightly shorter than mean solar seconds, and this characteristic was passed along to the atomic second, making the need for periodic leap seconds inevitable. However, even if the atomic second had been defined with respect to the mean solar second, there would still be a need for leap seconds, albeit a much smaller number. Leap seconds would still have been occasionally needed due to reason two, which is simply that the mean solar second is gradually getting longer because Earth's average rotational rate is gradually slowing down. The second reason is commonly 
cited as the chief reason for leap seconds, but it has had far less impact on the number of leap seconds that have occurred so far than the first reason.

\section{Commercial Cesium Clocks}

Commercial cesium clocks were available even before the publication of the Markowitz/Essen measurement in 1958, and several different models designed and manufactured in the United States were in use in laboratories prior to the adoption of the cesium definition in 1967. The availability of these commercial devices, as well as the measurement results and experience gained from their use, undoubtedly expedited the acceptance of the atomic definition of the SI second.

The first commercial cesium clock was introduced in October 1956, just slightly more than one year after the introduction of the NPL clock. Named the Atomichron, the clock was manufactured by the National Company of Malden, Massachusetts, and developed by a team led by Jerrold Zacharias of MIT [8, 37], an experienced researcher who had previously collaborated on early molecular beam experiments with Rabi [4] at Columbia University. About 50 Atomichrons were sold between 1956 and 1960, most of them to the U.S. military, with the first unit being delivered to the Naval Research Laboratory in Washington, D.C., in September 1956. The original models, numbers NC1001 and NC2001, were large devices, housed in an equipment rack that was about $0.6 \mathrm{~m}$ deep by $0.6 \mathrm{~m}$ wide, and about $2.1 \mathrm{~m}$ tall, and they weighed more than $350 \mathrm{~kg}$. The cesium beam tube was vertically mounted (Fig. 8) and nearly as tall as the equipment rack [8,38]. The resonance frequency of the first model was 9192631830 cycles/s $( \pm 10$ cycle/s), or $60 \mathrm{~Hz}$ higher than the yet-to-be published definition [37].

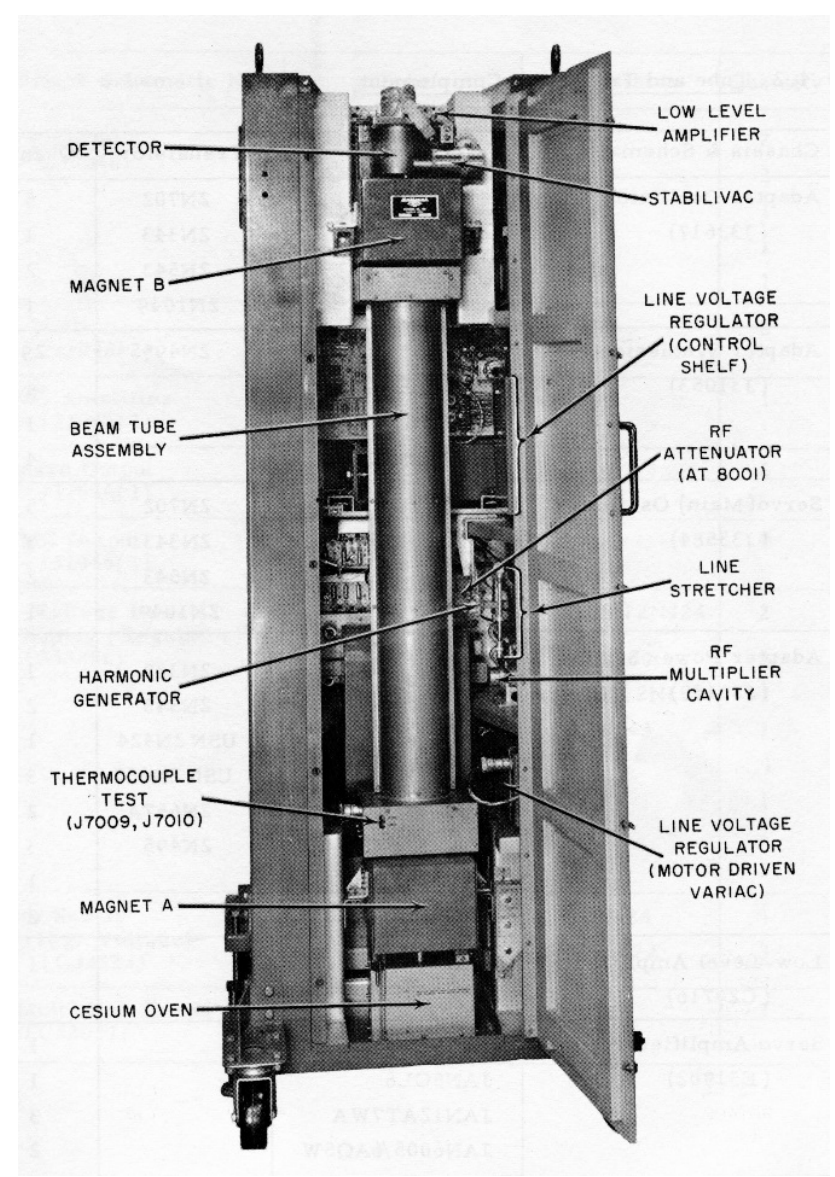

Fig. 8. Interior of Atomichron NC2001, showing vertical cesium beam tube (courtesy of Tom Van Baak, leapsecond.com). 
At least two Atomichrons were in operation at NBS in 1958-1959, where they were compared to the NBS standard to within parts in $10^{10}$ [23]. An additional two devices were transported to the United Kingdom and compared to Essen's clock at NPL with similar results [39]. Much smaller Atomichrons appeared later, produced mostly for the U.S. Air Force. However, the National Company ultimately failed due to financial difficulties and sold its assets, intellectual property, and trade names to Frequency Electronics, Inc., in 1969 [8, 38].

A far more successful line of cesium clocks was developed through the efforts of Leonard Cutler and his colleagues at the Hewlett-Packard Company in Santa Clara, California. Born in Los Angeles, California, in 1928, Cutler earned a Ph.D. from Stanford University and spent over four decades working for Hewlett-Packard and its successor, Agilent Technologies, where he designed or co-designed numerous frequency standards and clocks. During Cutler's tenure, Hewlett-Packard produced a series of cesium clocks that were reliable enough to run continuously for years and small enough to fit into standard equipment racks and were thus introduced into many calibration and metrology laboratories in the 1960s. The first of these clocks was the Hewlett-Packard 5060, introduced in 1964, some three years before the redefinition of the SI second (Fig. 9). The first cesium standard to feature all solid-state electronics, the length of its Ramsey cavity was just $12.4 \mathrm{~cm}$, and the device weighed less than $30 \mathrm{~kg}$ [38, 40]. By 1966, the 5060 had a specified accuracy of $1 \times 10^{-11}$ [21]. The 5060 was followed by the 5061, manufactured from 1967 until the early 1990s, and then by the 5071, which debuted in 1991. With an internal microprocessor and an improved cesium beam tube, the 5071 was more stable and accurate than all its predecessors [38]. It has a specified accuracy of about $20 \mathrm{~ns} /$ day $\left(2 \times 10^{-13}\right)$, and could easily be adjusted to keep time within a few nanoseconds per day (parts in $10^{14}$ ). Later manufactured by Agilent and Symmetricom and now manufactured by Microsemi, the 5071 continues to serve as the primary frequency and time standard at numerous laboratories.

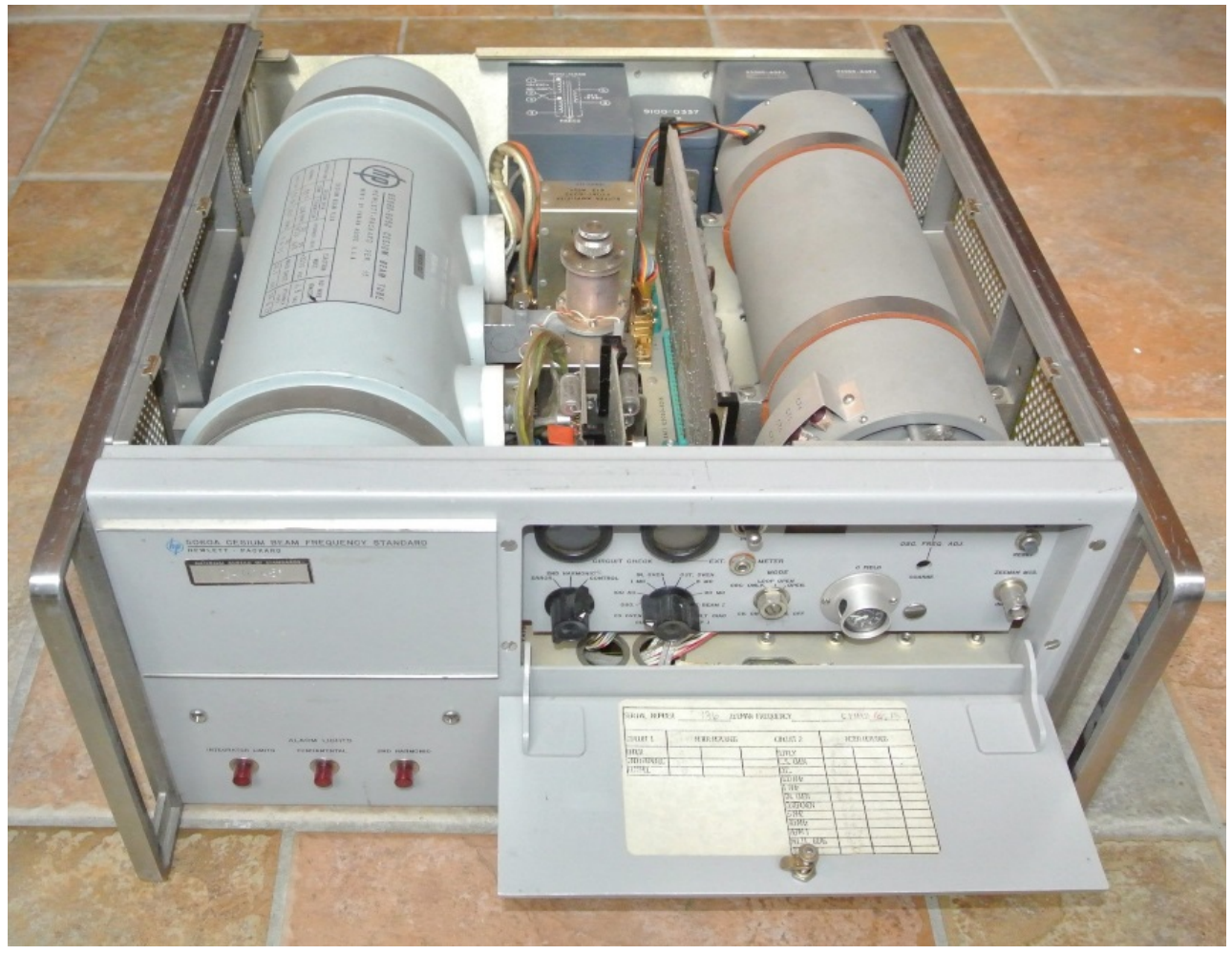

Fig. 9. Hewlett-Packard 5060A cesium beam atomic clock with cover removed showing beam tube on left (courtesy of Tom Van Baak, leapsecond.com). 


\section{Principles of a Cesium Clock}

The designs of cesium beam standards can vary significantly, but all designs, including those of the commercially available clocks still being sold in 2017 and the early cesium beam clocks built at NBS, NPL, and other laboratories, are based on principles that can be traced back to the seminal work of Rabi and Ramsey.

To understand how a cesium clock implements the SI definition of the second, consider that cesium is a complicated atom with $F=3$ and $F=4$ ground states (Fig. 10). Each atomic state is characterized not only by the quantum number $F$, but also by a second quantum number, $m_{\mathrm{F}}$, which can have integer values between $-F$ and $+F$. The splitting of the $F=3$ and $F=4$ states into the various $m_{\mathrm{F}}$ sublevels occurs in the presence of a magnetic field. There are 16 possible $m_{\mathrm{F}}$ sublevels in the ground state of cesium, but the frequency of the $|4,0\rangle \leftrightarrow|3,0\rangle$ hyperfine transition was the one chosen to define the second. The $|4,0\rangle \leftrightarrow$ $|3,0\rangle$ transition had several advantages that made it the best choice for clocks. There was a very low probability of a spontaneous transition occurring during the observation time. The transition was also relatively easy to detect with electronic systems that were already available when Rabi and others began their experiments. Perhaps most importantly, the transition was relatively insensitive to magnetic fields, so small magnetic fields near a cesium clock would have little effect on its frequency.

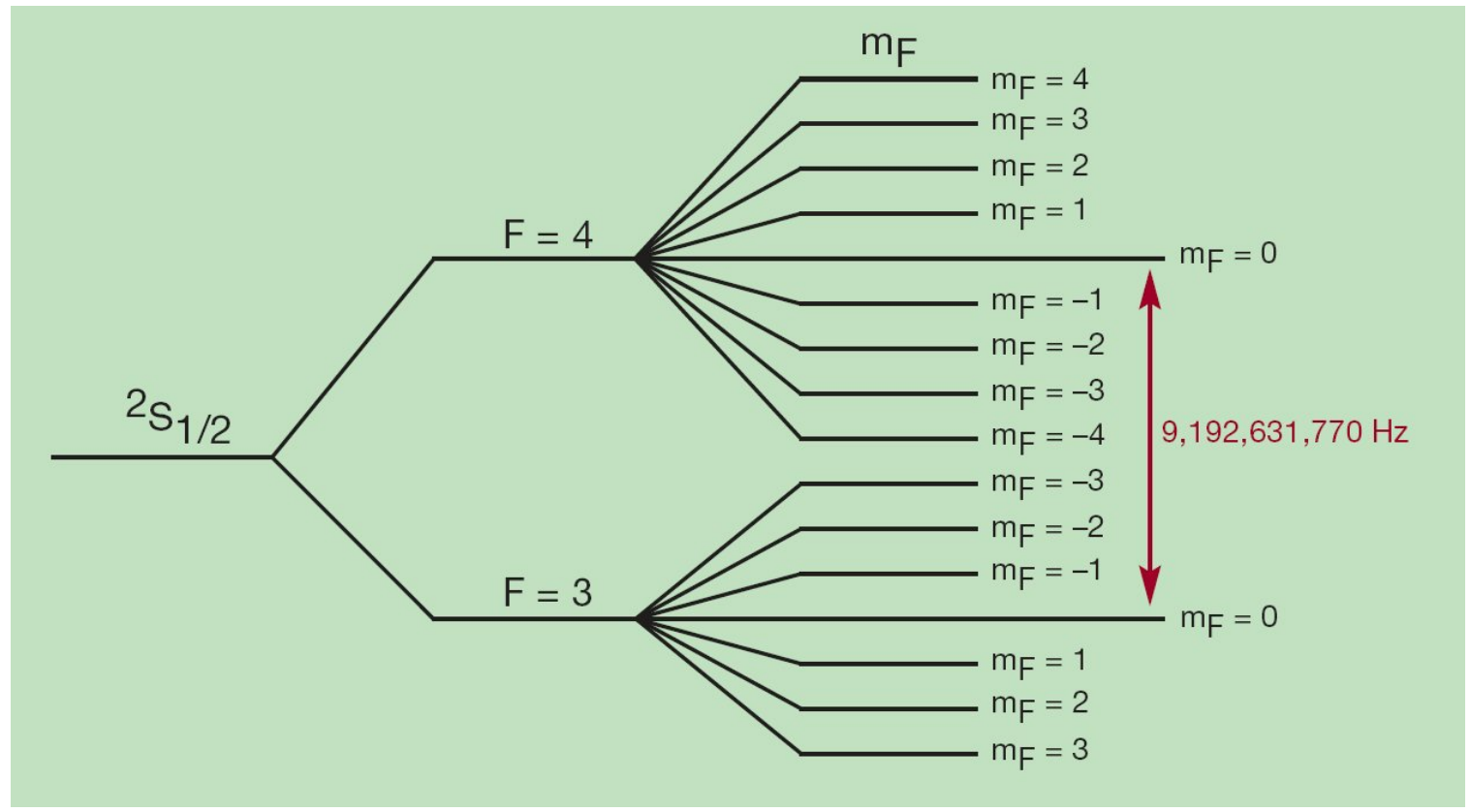

Fig. 10. The cesium clock transition.

Figure 11 provides a simplified schematic of a cesium beam clock. As shown on the left side of Fig. $11,{ }^{133} \mathrm{Cs}$ atoms are heated to a gaseous state in an oven. A beam of atoms emerges from the oven at a temperature near $100{ }^{\circ} \mathrm{C}$ and travels through a spatially varying magnetic field, where the beam is split into two atomic beams with different magnetic states that follow different trajectories. One beam is absorbed by the getter and is of no further interest. The other beam is deflected into the microwave interrogation cavity (i.e., Ramsey cavity), which exposes the atoms to microwaves in two spatially separate pulses.

While inside the Ramsey cavity, the cesium beam is exposed to a microwave signal. This signal is generated by a frequency synthesizer driven by a quartz oscillator. If this frequency exactly equals the cesium resonance frequency, the atoms will change their magnetic state (the desired $|4,0\rangle \leftrightarrow|3,0\rangle$ energy transition shown in Fig. 10) while inside of the Ramsey cavity. After leaving the cavity, the atoms pass through a second spatially varying magnetic field. These magnets direct only the atoms that changed state to the detector; the other atoms are directed to a getter and absorbed. The detector sends a feedback signal 
to a servo circuit that continually tunes the quartz oscillator so that the maximum number of atoms reaches the detector, thereby locking the frequency of the microwaves to the cesium hyperfine transition frequency. The process of keeping the quartz oscillator locked to the cesium frequency is analogous to carefully and continuously tuning a radio dial to ensure that the loudest and clearest signal is always heard [41, 42].

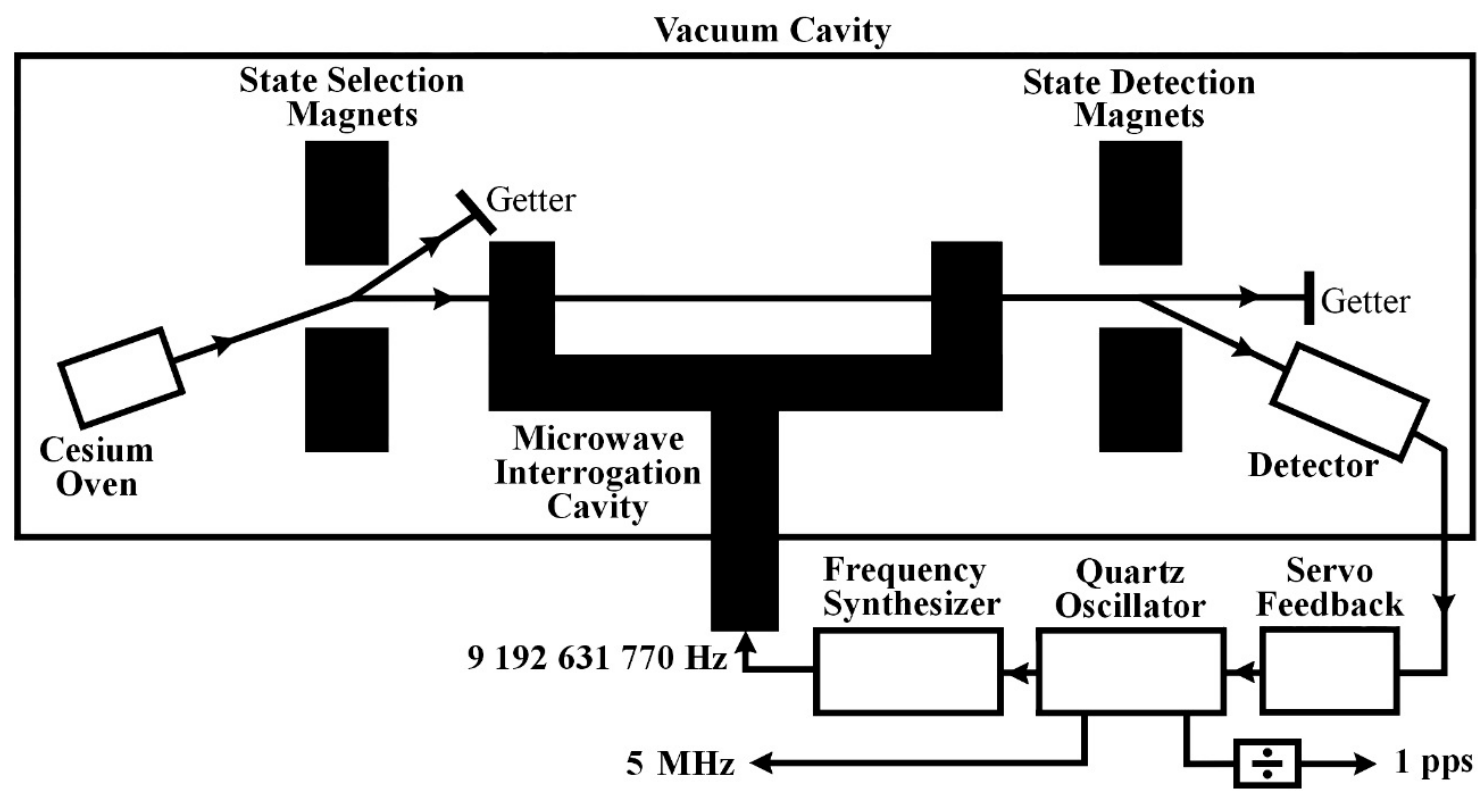

Fig. 11. Block diagram of a cesium-beam clock.

\section{Summary and Conclusion}

Countless contributions leading to the atomic definition of the SI second in 1967 were made by U.S. scientists and engineers, and so any historical account of this length cannot be comprehensive. The names of many individuals and organizations who helped to establish the modern era of atomic timekeeping have been regrettably omitted. This historical overview has briefly described the technology and noted the accomplishments of individuals, including Isidor Isaac Rabi, Sidney Millman, Polykarp Kusch, Norman Ramsey, Jerrold Zacharias, Harold Lyons, Jesse Sherwood, Richard Mockler, William Markowitz, and Leonard Cutler, and of organizations, including Columbia University, the Massachusetts Institute of Technology, Harvard University, the National Bureau of Standards, the U.S. Naval Observatory, the National Company, and the Hewlett-Packard Company. Their contributions, and the societal benefits of atomic timekeeping that we now enjoy because of their work, should not be forgotten.

\section{Acknowledgments}

The author thanks Elizabeth Donley and Tom Heavner of NIST for their review of this manuscript, and for many helpful comments and corrections. The author also thanks the six external peer reviewers whose comments and suggestions made the paper stronger. 


\section{References}

[1] Anonymous (1967) Resolution 1. 13th Conference Generale des Poids et Mesures (CGPM). http://www.bipm.org/en/CGPM/db/13/1/

[2] Thomson W, Tait P (1879) Elements of Natural Philosophy (Cambridge University Press, Cambridge, United Kingdom), pp 6162.

[3] Snyder W (March 1973) Lord Kelvin on atoms as fundamental natural standards (for base units). IEEE Transactions on Instrumentation and Measurement IM-22:99. https://doi.org/10.1109/TIM.1966.4313501

[4] Rabi I, Zacharias J, Millman S, Kusch P (February 1938) A new method of measuring nuclear magnetic moment. Physical Review 53:318. https://doi.org/10.1103/PhysRev.55.526

[5] Ramsey N (1956) Molecular Beams (Clarendon Press, Oxford).

[6] Ramsey N (September-October 1983) History of atomic clocks. Journal of Research of the National Bureau of Standards 88:301-320. http://dx.doi.org/10.6028/jres.088.015

[7] Laurence W (1945) Cosmic pendulum for clock planned. New York Times, January 21, 1945, p 34.

[8] Forman P (July 1985) Atomichron ${ }^{\mathrm{TM}}$ : The atomic clock from concept to commercial product. Proceedings of the IEEE 73:11811204. https://doi.org/10.1109/PROC.1985.13266

[9] Millman S, Kusch P (September 1940) On the radiofrequency spectra of sodium, rubidium, and caesium. Physical Review 58:438-445. https://doi.org/10.1103/PhysRev.58.438

[10] Forman P (1985) The first atomic clock program: NBS, 1947-1954. Proceedings of the 1985 Precise Time and Time Interval Meeting (PTTI). Smithsonian, Institution, Washington, D.C., pp 1-17.

[11] National Bureau of Standards (January 1949) The Atomic Clock: An Atomic Standard of Frequency and Time. U.S. Department of Commerce, Washington, D.C., National Bureau of Standards Technical Report 1320.

[12] Lyons H (December 1949) The atomic clock. Instruments 22:133-135.

[13] Lyons H (November 1952) Spectral lines as frequency standards. Annals of the New York Academy of Sciences 55:831-871. https://doi.org/10.1111/j.1749-6632.1952.tb26600.x

[14] Lyons H, Huston B (1955) Atomic Clock. United States Patent 2,699,503 (applied for April 30, 1949; granted January 11, 1955).

[15] Lyons H (January 1950) Microwave frequency dividers. Journal of Applied Physics 21:59-60. https://doi.org/10.1063/1.1699421

[16] Lyons H (April 1950) The atomic clock: A universal standard of frequency and time. American Scholar 19:159-168. http://www.jstor.org/stable/41205288

[17] Sherwood J, Lyons H, McCracken R, Kusch P (1952) High frequency lines in the hfs spectrum of cesium. Bulletin of the American Physical Society 27:43.

[18] N. Ramsey (June 1950) A molecular beam resonance method with separated oscillating fields. Physical Review 78:695-699. https://doi.org/10.1103/PhysRev.78.695

[19] Lyons H (August 8, 1952) Spectral Lines as Frequency Standards. U.S. Department of Commerce, Washington, D.C., National Bureau of Standards Report 1848.

[20] Snyder W, Bragaw C (October 1986) Achievement in Radio: Seventy Years of Radio Science, Technology, Standards, and Measurement at the National Bureau of Standards. U.S. Department of Commerce, Washington, D.C., National Bureau of Standards Special Publication 555.

[21] Beehler R (June 1967) A historical review of atomic frequency standards. Proceedings of the IEEE 55:792-805. https://doi.org/10.1109/PROC.1967.5692

[22] Mockler R, Beehler R, Snider C (September 1960) Atomic beam frequency standards. IRE Transactions on Instrumentation I9:120-132.

[23] Mockler R, Beehler R, Barnes J (October 1959) An Evaluation of a Cesium Beam Frequency Standard. U.S. Department of Commerce, Washington, D.C., National Bureau of Standards Report 6075.

[24] Beehler R, Glaze D (March-June 1966) The performance and capability of cesium beam frequency standards at the National Bureau of Standards. IEEE Transactions on Instrumentation and Measurement 15:48-55. https://doi.org/10.1109/TIM.1966.4313501

[25] Henderson D (June 2005) Essen and the National Physical Laboratory’s atomic clock. Metrologia 42:S4-S9. https://doi.org/10.1088/0026-1394/42/3/S02

[26] Essen L, Parry J (August 1955) An atomic standard of frequency and time interval: A caesium resonator. Nature 176:280-281.

[27] Audoin C, Guinot B (2001) The Measurement of Time: Time, Frequency and the Atomic Clock (Cambridge University Press, Cambridge, United Kingdom).

[28] McCarthy D, Seidelmann P (2009) Time: From Earth Rotation to Atomic Physics (Wiley-VCH, Weinheim, Germany).

[29] Markowitz W (March 1954) Photographic determination of the Moon's position, and applications to the measure of time, rotation of the Earth, and geodesy. The Astronomical Journal 59:69-73. https://doi.org/10.1086/106956

[30] Bullard E (August 1955) Definition of the second of time. Nature 176:282.

[31] Leschiutta S (June 2005) The definition of the 'atomic' second. Metrologia 42:S10-S19. https://doi.org/10.1088/00261394/42/3/S03

[32] Markowitz W, Glenn Hall R, Essen L, Parry J (August 1958) Frequency of cesium in terms of ephemeris time. Physical Review Letters 1(3):105-107. https://doi.org/10.1103/PhysRevLett.1.105

[33] Markowitz W (December 1962) The atomic time scale. IRE Transactions on Instrumentation I-11(3):239-242. https://doi.org/10.1109/IRE-I.1962.5006637

[34] Tipson R (November 1961) Sun-time replaced by atomic clocks. The Capital Chemist 11:255-256.

[35] Terrien J (October 1967) A major step towards the redefinition of the second, the unit of time in the International System of Units. Metrologia 3(4):130. https://doi.org/10.1088/0026-1394/3/4/007 
[36] Itano W, Lewis L, Wineland D (February 1982) Shift of ${ }^{2} S_{1 / 2}$ hyperfine splittings due to blackbody radiation. Physical Review $A$ 25:1233-1235.

[37] Anonymous (1957) Atomichron—World's most accurate clock. Radio and Television News, January 1957, p 63.

[38] Cutler L (June 2005) Fifty years of commercial caesium clocks. Metrologia 42:S90-S99. https://doi.org/10.1088/0026$1394 / 42 / 3 / \mathrm{S} 10$

[39] McCoubrey A (1958) Results of comparison: Atomichron-British cesium beam standard. Proceedings of the 12th Annual Frequency Control Symposium, pp 648-664. https://doi.org/10.1109/IRE-I.1958.5006789

[40] Bagley A, Cutler L (1964) A modern solid-state portable cesium beam frequency standard. Proceedings of the 18th Annual Frequency Control Symposium, pp 344-365. https://doi.org/10.1109/FREQ.1964.199548

[41] Itano W, Ramsey N (July 1993) Accurate measurement of time. Scientific American 269:56-65. https://doi.org/10.1038/scientificamerican0793-56

[42] Major F (1998) The Quantum Beat: The Physical Principles of Atomic Clocks (Springer-Verlag, New York).

About the author: Michael A. Lombardi is a staff member in the services group of the Time and Frequency division of the National Institute of Standards and Technology (NIST) in Boulder, Colorado. He is the project manager for the division's remote frequency and time calibration services and serves as the division's quality manager. The National Institute of Standards and Technology is an agency of the U.S. Department of Commerce. 